CASE REPORT

\title{
Giant Right Atrium or Ebstein Anomaly?
}

\author{
Hacer Kamalı ${ }^{1}$, Abdullah Erdem ${ }^{1 *}$, Cengiz Erol ${ }^{2}$ and Atf Akçevin ${ }^{3}$ \\ ${ }^{1}$ Department of Pediatric Cardiology, Istanbul Medipol University, Turkey \\ ${ }^{2}$ Department of Radiology, Istanbul Medipol University, Turkey \\ ${ }^{3}$ Department of Cardiovascular Surgery, Istanbul Medipol University, Turkey
}

*Corresponding author: Abdullah Erdem, Associate Professor of Pediatric Cardiology, Department of Pediatric Cardiology, Istanbul Medipol University, Istanbul, Turkey, Tel: +905-0577-05805, Fax: +90212467064, E-mail: drabdullaherdem@ hotmail.com

\begin{abstract}
Many acquired and congenital pathologies could cause enlargement of Right Atrium (RA). In rare pathologies we couldn't find an obvious reason for enlargement of RA. Sometimes it leads to misdiagnosis if we try to explain an unknown pathology with well known pathology. In this article we describe a woman who presented with giant enlargement of the RA misdiagnosed as Ebstein anomaly. Cardiac MRI is highly helpful for differential diagnosis of these kinds of atrial pathologies. Although it is rare, giant RA should be considered in differential diagnosis of huge RA without an obvious cause.
\end{abstract}

\section{Keywords}

Giant right atrium, Ebstein anomaly

\section{Introduction}

There are several reasons that could cause tricuspid regurgitation and Right Atrium (RA) dilatation. Primary tricuspid valve pathologies; both acquired and congenital may dilate RA proportional to severity of pathologies $[1,2]$. Also pathologies related with Right Ventricle (RV) could cause RA dilatation. Congenital malformations of RA and coronary sinus are also infrequently encountered and only sporadic cases have been presented $[1,2]$. Idiopathic dilatation of the RA was also described in literature $[3,4]$. In this article we describe a woman who presented with giant enlargement of the RA misdiagnosed as Ebstein anomaly.

\section{Case}

A 24-year-old woman applied to the cardiology de- partment for exercise intolerance. She had been diagnosed and followed up as Ebstein anomaly for ten years. Her heart rate was $75 / \mathrm{min}$ with normal jugular venous pressure. Findings of lung examination were normal. Auscultation of heart revealed a 2/6 systolic murmur on the lower left edge of the sternum. On abdominal examination, there was no organomegaly. Arterial Oxygen Saturation (SpO2) was 98\%. Electrocardiography (ECG) revealed absence of atrial fibrillation, the rhythm was sinus but there was right axis deviation and right bundle branch block (Figure 1). There was a significant cardiomegaly (cardiothoracic ratio: 0.7 ) on telecardiography (Figure 2). Echocardiography demonstrated a severely dilated RA measuring $96 \mathrm{~mm} \times 135 \mathrm{~mm}$ in four chamber view (Figure 3 ) and RV pressure was $40 \mathrm{mmHg}$ indirectly calculated by tricuspid valve regurgitation. There was severe tricuspid regurgitation. Since there was no evidence of apical displacement of the tricuspid septal leaflet, Ebstein's anomaly was not considered.

Cardiac MRI confirmed that tricuspid valve leaflets were of normal structure. Atrium volume was calculated $617 \mathrm{ml}$ and highly dilated (Figure 4a and Figure 4b) and there was severe tricuspid insufficiency. The location and orientation of the tricuspid valve in relation to the mitral valve were normal with no evidence of apical displacement to suggest Ebstein's anomaly. Right ventricle wall motion was normal and RV ejection fraction was $63 \%$.

Findings of echocardiography and cardiac MRI excluded Ebstein anomaly. Giant RA was appropriate di-

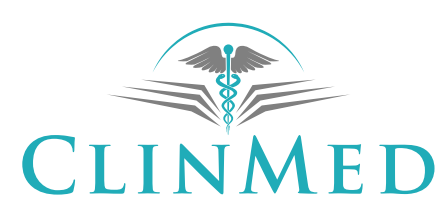

INTERNATIONAL LIBRARY
Citation: Kamalı H, Erdem A, Erol C, Akçevin A (2017) Giant Right Atrium or Ebstein Anomaly?. Int J Clin Cardiol 4:104. doi.org/10.23937/2378-2951/1410104

Received: August 12, 2017; Accepted: October 07, 2017; Published: October 09, 2017

Copyright: (c) 2017 Kamalı H, et al. This is an open-access article distributed under the terms of the Creative Commons Attribution License, which permits unrestricted use, distribution, and reproduction in any medium, provided the original author and source are credited. 


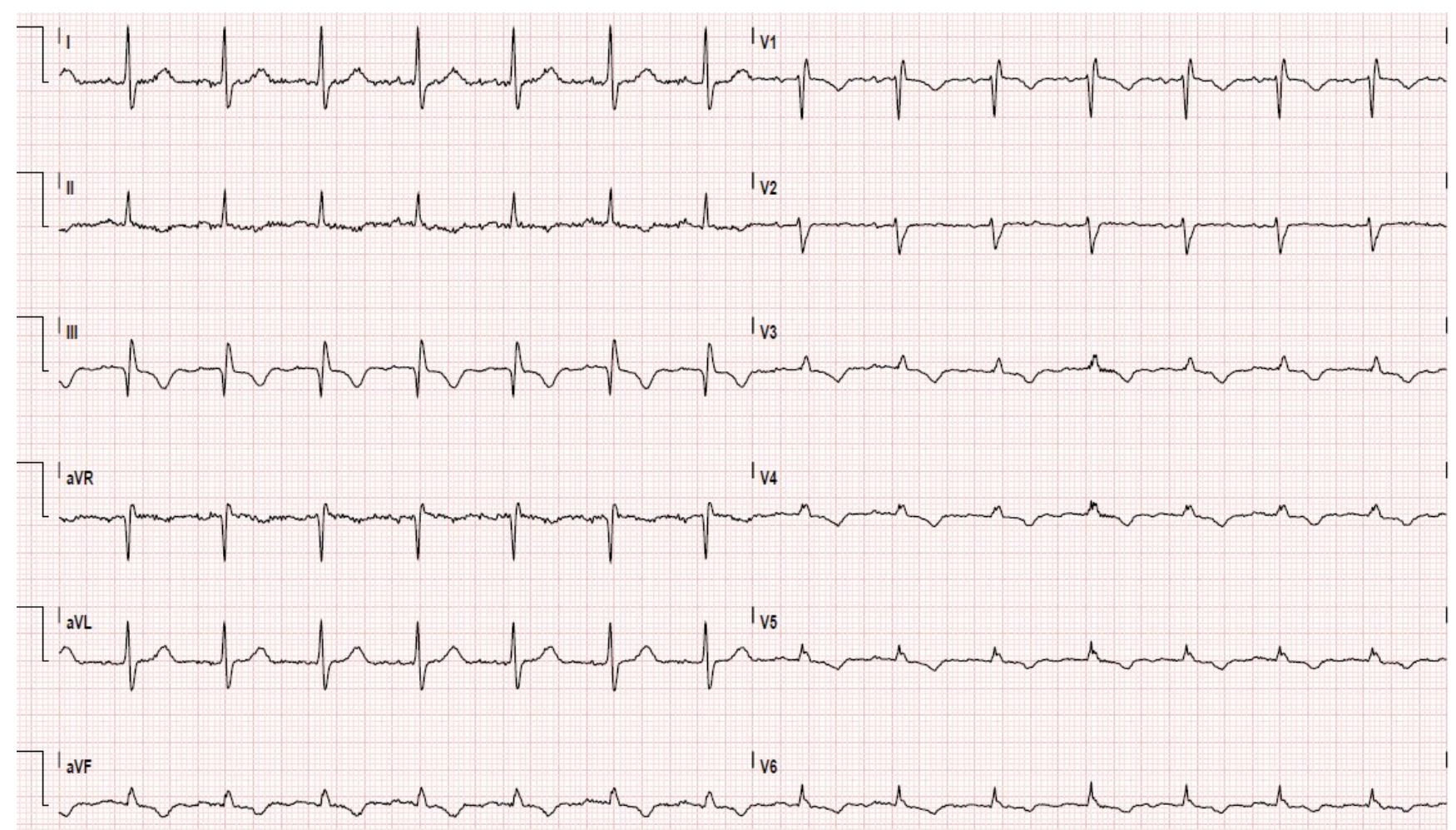

Figure 1: ECG demonstrates right axis deviation and right bundle branch block.

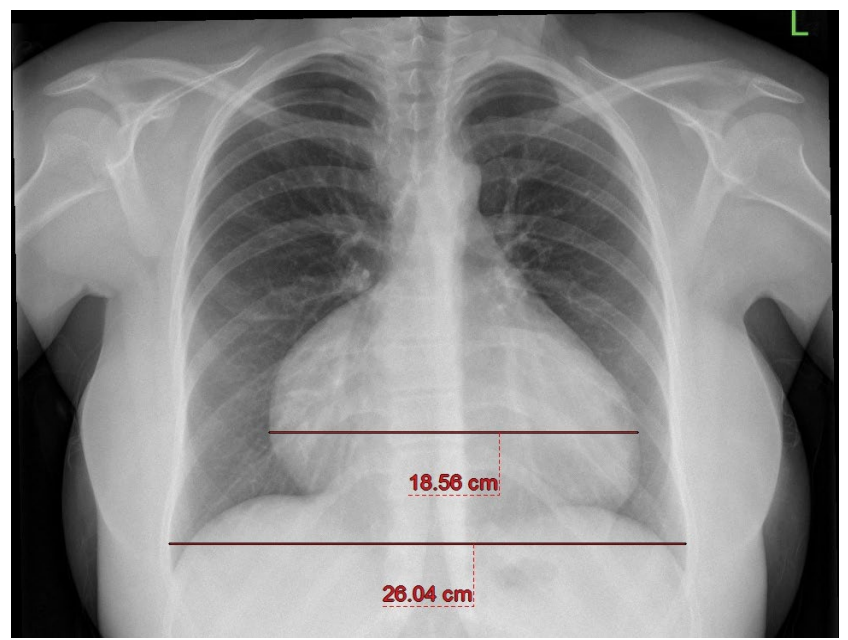

Figure 2: Telecardiography shows significant cardiomegaly.

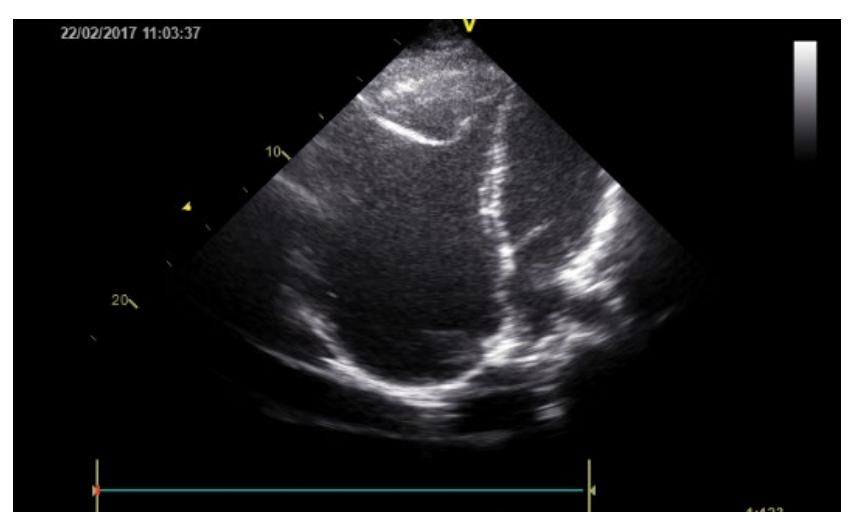

Figure 3: Transthoracic echocardiogram in the four-chamber apical view demonstrating a massively dilated right atrium.

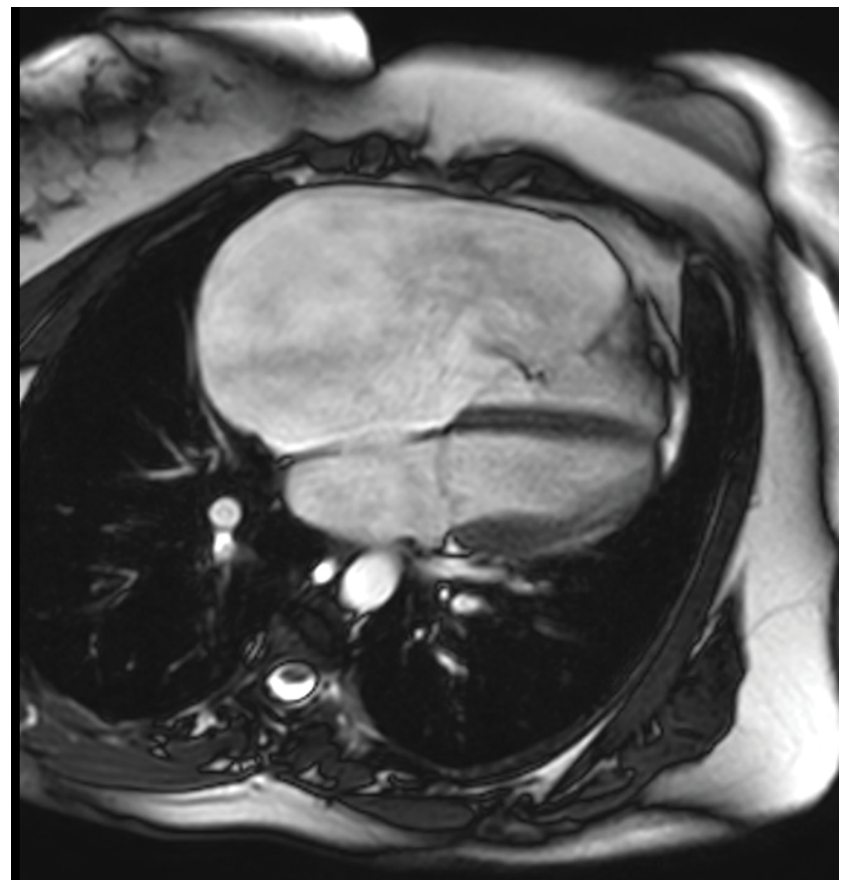

Figure 4a: Cardiac MR four chamber image demonstrates a markedly enlarged right atrium. Note the normal relation of the mitral and tricuspid valves.

agnosis. And pathology caused severe tricuspid regurgitation and the patient was symptomatic. Open-heart surgery for reduction atrioplasty and tricuspid ring annuloplasty was planned for the patient.

Surgical exploration showed right atrium filling the whole right thoracic cavity. The tricuspid annulus was severely dilated and its chordae were thin. Severe TV 


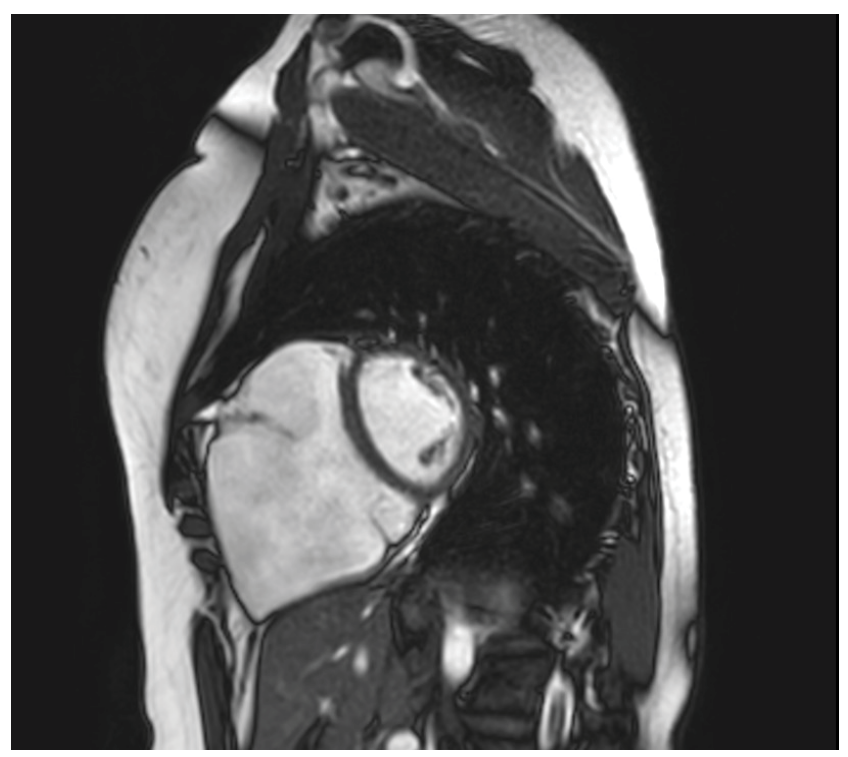

Figure 4b: Short axis image again demonstrates the markedly enlarged right atrium.

annular dilation was present with normally positioned leaflets. Leaflet quality was normal. The giant atrium wall was very thin and the Right Coronary Artery (RCA) was transmural. A $32 \mathrm{~mm}$ Medtronik 3D ring (Contour 3D Annuloplasty System (Medtronic, Minneapolis, MN, USA) was implanted and reduction atrioplasty was performed. Right coronary artery was protected. Patient was weaned off cardiopulmonary bypass with minimal inotropic support and extubated in the early postoperative period. Further postoperative course was uneventful.

Histological assessment of the right atrial wall showed weakening and atrophy of the muscle fibre layer and fibrous thickening of the endocardium and visceral pericardium. The appearance was non-diagnostic. Myocardial cells showed mild oedema.

Postoperative echocardiography demonstrated that tricuspid regurgitation was minimal without any stenosis; RA was somewhat smaller (RA $65 \mathrm{~mm} \times 55 \mathrm{~mm}$ ).

Her symptoms disappeared in her follow-up and effort capacity was fully normalized.

\section{Discussion}

There are several reasons that could cause tricuspid regurgitation and RA dilatation. Primary tricuspid valve pathologies; both acquired and congenital may dilate RA proportional to severity of pathologies. Also pathologies related with RV could cause RA dilatation. Therefore chronic pulmonary disease, severe mitral valvular pathology leading to pulmonary hypertension, and pulmonary emboli or infarction should be considered in diagnosis of dilated RA. Diastolic dysfunction of RV, restrictive cardiomyopathy and in some cases, constrictive pericarditis should also be considered in differential diagnosis.

Congenital malformations of RA and coronary sinus are also infrequently encountered and only sporadic cases have been presented [1]. The enlarged RA is defined as "paper thin" and translucent in several patients $[1,3]$. This pathologic appearance can imitate strongly to the description of cases so called "Parchment Heart" or Uhl's anomaly [3]. But in Uhl's anomaly there is evident myocardial hypoplasia or aplasia comprising one or more cardiac chambers.

Giant RA can imitate Ebstein anomaly in some patients. The main morphological characteristic of Ebstein anomaly is the movement of the posterior and/or septal valve leaflets of the tricuspid valve downward into the $R V$, leading to RV divided into two regions. The area involved with the malformation; "atrialized RV; and the normal area constitute the functional RV [5-7]. Cardiac MRI provides quantitative measurement of right atrial and ventricular size and systolic function even in the presence of significant distortion of right ventricular anatomy.

For our patient cardiac MRI confirmed that tricuspid leaflets were of normal structure. Right atrium was highly dilated and there was severe tricuspid insufficiency. The location and orientation of the tricuspid valve in relation to the mitral valve were normal with no evidence of apical displacement to suggest Ebstein anomaly. Right ventricle wall motion and RV function was normal. Findings of echocardiography and cardiac MRI excluded Ebstein anomaly.

Idiopathic dilatation of the RA was first described by Bailey [4]. Only there are few case reports and limited study on this subject [8-11]. The largest description of the RA was reported by Demarco and Bolero, who mentioned "atriomegaly" in a child where the RA volume was reported to be $900 \mathrm{ml}$ [12]. The reported another case was an adult in the setting of desmin-related restrictive cardiomyopathy and had an estimated RA volume of $461 \mathrm{ml}$ [13].

In this pathology clinical presentations depend on the type of RA malformation. Approximately half of the patients with giant RA are asymptomatic at time of diagnosis. Commonly reported symptoms are shortness of breath, fatigue, chest pain, right heart failure, palpitations and syncope. Sudden death was also declared in a 16- year-old boy who rejected surgical treatment [14].

Our patient had complaints of intermittent shortness of breath and fatigue. Patients can also submit with atrial arrhythmias. Our patient did not have any atrial arrhythmia but she has right bundle branch block on ECG.

Enlarged RA can induce symptoms related to compression of intrapericardial structures such as jugular engorgement, edema and hepatomegaly. In our patient neither clinical findings nor MR showed evidence of compression. Often, functional tricuspid regurgitation accompanies the giant RA, secondary to chamber enlargement, as noted in our case. 
Surgical reduction atrioplasty is necessary in most cases $[3,8]$, significant tricuspid regurgitation require tricuspid annuloplasty. Treatment of asymptomatic patients is controversial. We found it appropriate to perform an operation because our patient described fatigue and exercise intolerance.

The enlarged RA can be damaged during sternotomy and the whole operating team was aware of this complication. We did not have any complications during the operation. Anticoagulation is necessary because of high risk of developing thrombus and atrial arrhythmia. Therefore we prescribed anticoagulation to our patient.

It is not known why dilation of the RA occurs. The causative mechanism might be a partial loss of atrial muscular fibers with progressive atrial enlargement. The optimal therapeutic approach is controversial. Most authors suggest surgery only in symptomatic patients. The optimal approach to these patients is not well established.

\section{Conclusion}

In conclusion, in patients with very large RA to diagnose a patient as an Ebstein anomaly, we should be sure that the patient fulfills the criteria of this anomaly. Cardiac MRI is highly helpful for differential diagnosis of these kinds of atrial pathologies. Although it is rare, giant RA should be considered in differential diagnosis if there is no reasonable explanation for huge atrial enlargement.

\section{Conflicts of Interest}

We declare that we have no conflict of interest. We also declare that the case is original and is not submitted anywhere we give all the rights to journal for publishing of this article.

\section{References}

1. Binder TM, Rosenhek $R$, Frank $H$, Gwechenberger $M$, Maurer G, et al. (2000) Congenital malformations of the right atrium and the coronary sinus: An analysis based on 103 cases reported in the literature and two additional cases. Chest 117: 1740-1748.

2. Guiraudon GM, Guriraudon CM, Klein GJ, Sharma AD, Yee $R$ (1988) The coronary sinus diverticulum: A pathologic entity associated with the Wolff-Parkinson-White Syndrome. Am J Cardiol 62: 733-735.

3. Sheldon WC, Johnson CD, Favaloro RG (1969) Idiopathic enlargement of the right atrium. Report of four cases. Am J Cardiol 23: 278-284.

4. Bailey CP (1955) Surgery of the heart. Lea \& Febiger Publisher, Philadelphia, 413.

5. Dearani JA, Danielson GK (2000) Congenital Heart Surgery Nomenclature and Database Project: Ebstein's anomaly and tricuspid valve disease. Ann Thorac Surg 69: S106-S117.

6. Dearani JA, Danielson GK (2003) Ebstein's anomaly of the tricuspid valve. In: Mavroudis C, Backer CL, Pediatric Cardiac Surgery. (3 ${ }^{\text {rd }}$ edn), PA: Mosby, Philadelphia, 524-536.

7. Attenhofer Jost $\mathrm{CH}$, Connolly HM, Dearani JA, Edwards WD, Danielson GK (2007) Ebstein's anomaly. Circulation 115: 277-284.

8. Kishore N, Ravi, Gowda N, Devananda (2005) Giant right atrium--a rare case report. Ann Card Anaesth 8: 58-60.

9. Ariyarajah V, Soni A, Morris A (2008) A Giant right atrium in an adult. Echocardiography 25: 1121-1123.

10. Kurz DJ, Oechslin EN, Kobza R, Jenni R (2004) Idiopathic enlargement of the right atrium: 23-year follow up of a familial cluster and their unaffected relatives. Heart 90: 1310-1314.

11. Gomes S, Wolfenden H, Lambros J (2012) Giant right atrium in an adult: Case report of a rare condition. Heart Lung Circ 21: 50-52.

12. De Marco S, Bollero E (1967) Isolated right atriomegaly. Boll Soc Ital Cardiol 12: 16-22.

13. Hager S, Mahrholdt H, Goldfarb LG, Goebel HH, Sechtem $U$ (2006) Images in cardiovascular medicine. Giant right atrium in the setting of desmin-related restrictive cardiomyopathy. Circulation 113: e53-e55.

14. Tenckhoff L, Stamm SJ, Beckwith JB (1969) Sudden death in idiopathic (congenital) right atrial enlargement: postmortem findings and review of cases. Circulation 40: 227-235. 